\title{
RDUS
}

Revue de DROIT

UNIVERSITÉ DE SHERBROOKE

Titre : $\quad$ LES ASSURANCES ET LES CAUTIONNEMENTS COMME PROTECTION CONTRE LES MALFAÇONS DANS LA CONSTRUCTION

Auteur(s) : $\quad$ Christianne DUBREUIL

Revue : $\quad$ RDUS, 1991-1992, volume 22, numéro 2

Pages: $\quad 343-368$

ISSN : $\quad 0317-9656$

Éditeur : $\quad$ Université de Sherbrooke. Faculté de droit.

URI : $\quad$ http://hdl.handle.net/11143/13425

DOI : https://doi.org/10.17118/11143/13425 
Page vide laissée intentionnellement. 


\section{LES ASSURANCES ET LES CAUTIONNEMENTS COMME PROTECTION CONTRE LES MALFAÇONS DANS LA CONSTRUCTION}

par Christianne DUBREUIL

Les assurances contre les malfaçons, dans le domaine de la construction, sont peu utilisées. Certes, les architectes bénéficient à cet égard d'une assurance de responsabilité professionnelle. Quant aux entrepreneurs, les polices d'assurance contiennent des exclusions concernant les malfaçons causées par un défaut dans la main-d'oeuvre. Ces exclusions ont été interprétées largement par les tribunaux alors que les tempéraments à ces exclusions l'ont été de façon restrictive. La Cour d'appel du Québec a fait preuve de plus d'ouverture en rejetant cette tendance et en adoptant une interprétation de la clause d'exclusion pour défaut de la main-d'oeuvre plus conforme aux règles d'interprétation des contrats d'assurance. Les cautionnements protègent dans une certaine mesure les consommateurs contre les erreurs des ouvriers, mais il s'agit d'une garantie qui comporte ses difficultés de mise en oeuvre, d'une part il faut poursuivre l'entrepreneur avant de réclamer à la caution et d'autre part cette dernière n'entrera en jeu qu'en cas de défaut.

Les malfaçons sont un risque assurable et l'assurance a un rôle $\grave{a}$ jouer dans ce domaine. En effet, les cautionnements ne protègent pas l'entrepreneur, ce dernier étant l'ultime débiteur. De plus, l'assurance offre une garantie plus souple et facile à administrer pour le consommateur.

*. Professeure à la Faculté de droit de l'Université de Montréal. 

dans la construction

In the field of construction contracts, insurance is rarely taken out against defects. Architects, of course, are covered by professional liability insurance. For building contractors however, insurance policies usually stipulate exclusions regarding defects caused by faulty workmanship. Although these exclusions have generally been broadly interpreted by the courts, attenuations to such exclusions have been given a narrow interpretation. The Quebec Court of Appeal has shown more openness in rejecting this tendency by adopting an interpretation of the exclusion clause for defects in workmanship more in keeping with the ordinary rules pertaining to the interpretation of insurance policies. To a certain extent, suretyships can protect consumers against faulty workmanship, but this type of protective measure is somewhat difficult to exercise since the contractor must be sued before a claim may be made upon the surety and the suretyship takes effect only in cases of default.

Defects constitute an insurable risk and insurance obviously can have a role to play in this area. Suretyships simply do not adequately protect the contractor since the latter remains the ultimate debtor. In addition, insurance provides a guarantee which is more flexible as well as easier for the consumer to exercise. 


\section{SOMMAIRE}

INTRODUCTION

CHAPITRE PRÉLIMINAIRE: Les différents fondements de responsabilité ........ 348

\section{PARTIE I - LES ASSURANCES CONTRE LES MAL-}

FAÇONS $\ldots \ldots \ldots \ldots \ldots \ldots \ldots \ldots$

A $\quad-\quad$ Les diverses assurances de responsabilité $\ldots \quad 350$

1: Les architectes et les ingénieurs . . . . ... 352

2: $\quad$ Les entrepreneurs . . . . . . . . . 353

- $\quad$ les types de contrat $\ldots \ldots \ldots \ldots \ldots .353$

- les exclusions ............. 355

- le tempérament à ces exclusions . . . 356

B $\quad-\quad$ Critique des protections d'assurance $\ldots \ldots \ldots 358$

PARTIE II - LES CAUTIONNEMENTS . . . . . . . 361

A - Les cautionnements en tant que garanties contre les malfaçons .............. 361

B - Critique du cautionnement en tant que garantie $\ldots \ldots \ldots \ldots \ldots \ldots \ldots \ldots \ldots$

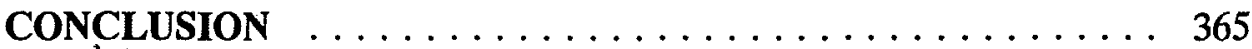




\section{INTRODUCTION}

L'assurance contre les malfaçons dans le domaine de la construction est à peu près inexistante. Est-ce la crainte que la négligence soit avalisée ou que l'assuré bénéficie de ses propres actes fautifs ${ }^{1}$ ? En effet, certains diront qu'elle serait une prime à l'incompétence voire à la négligence de la part des différents intervenants. Pourtant, il est de l'essence même de l'assurance de protéger l'assuré non seulement contre les événements externes, mais aussi contre ses propres fautes et faiblesses, sinon l'assurance de responsabilité civile n'aurait plus sa raison d'être. Certes, l'assurance de responsabilité est offerte et utilisée par les professionnels tels les architectes et les ingénieurs. Par contre, dans le cas des entrepreneurs, elle est largement insuffisante sinon totalement absente de certaines garanties. Qu'il suffise de mentionner tout de suite qu'il n'existe pas d'assurance pour les vices cachés ou pour les malfaçons qui apparaissent lorsque l'ouvrage est terminé, ces événements étant plutôt garantis par des contrats de cautionnement.

Il est important de spécifier que dans le domaine de la construction, il n'existe pas au Québec d'obligation de souscrire une assurance ou un cautionnement dans le cadre des activités professionnelles ${ }^{2}$. Les associations professionnelles regroupant les architectes ${ }^{3}$ et les ingénieurs ${ }^{4}$ font présentement pression sur le gouvernement québécois afin qu'une législation soumette l'obtention du permis d'exercer la profession à la souscription d'une assurance de responsabilités. Quant aux entrepreneurs, ils ne sont pas tenus de souscrire à des contrats de cautionnement pour garantir leurs ouvrages contre les vices cachés et les malfaçons si ce n'est que lorsque le cocontractant l'exige; par exemple, en matière de construction d'édifices publics ou commerciaux et lors

1. George J. COUCH, Cyclopedia of Insurance Law, $2^{\complement}$ éd. vol. 11, The Lawyers Cooperative Publishing Co., 1982, p. 437 cité dans: Groupe Desjardins Assurances générales c. L. Breton \& Fils (La Sarre) Ltée, [1989] R.R.A. 221 (C.A.).

2. Il est intéressant de noter que la France impose une obligation d'assurance dans le domaine de la construction: Code des assurances, art. 241.1,241.2, 242.1 et 242.2, Loi $n^{\circ} 78-12$ du 4 janvier 1978 et Loi no 89-1014 du 31 décembre 1989.

3. L'Ordre des architectes est formé en vertu de la Loi sur les architectes, L.R.Q., c. A-21.

4. L'Ordre des ingénieurs est formé en vertu de la Loi sur les ingénieurs, L.R.Q., c. I-9.

5. Le permis d'exercice est émis par les corporations professionnelles selon les conditions prescrites par la loi et les règlements: Code des professions, L.R.Q., c. C-26, art. 40. 
de projets gouvernementaux, les cautionnements et les assurances sont habituellement une exigence préalable à la conclusion du contrat ${ }^{6}$.

Nous verrons successivement les assurances et les cautionnements qui ont cours en tant que garanties pendant les travaux et ceux qui s'appliquent après la réception par le maître d'ouvrage. Nous discuterons des diverses possibilités qui peuvent s'offrir dans ce domaine afin que les constructeurs tout autant que les maîtres d'ouvrage soient mieux protégés.

\section{CHAPITRE PRÉLIMINAIRE: Les différents fondements de res- ponsabilité}

Il n'est pas de notre propos de discuter des différents aspects de la responsabilité des constructeurs. Nous devons néanmoins faire les distinctions pertinentes afin d'étudier adéquatement les risques couverts par les contrats d'assurance offerts sur le marché québécois.

Selon l'article 1688 du Code civil du Bas Canada', l'entrepreneur et l'architecte qui a surveillé les travaux $^{8}$ sont responsables de la ruine du

6. Lorsque des travaux sont exécutés pour l'État, ce dernier exigera des sûretés: Loi sur le ministère des Transports, LR.C. (1985), c. T-18, art. 10, Loi sur les travaux publics, L.R.C. (1985), c. P-38, art. 16, Loi sur l'administration financière, L.R.Q., c. A-6, Règlement sur les contrats de construction du gouvernement, R.R.Q., 1981, c. A-6, r. 7, art. 14(b)(i). Il revient au maître d'ouvrage d'exiger de l'entrepreneur des garanties: Louise POUDRIER-LEBEL, «Les cautionnements des contrats de construction: réduire les risques en faisant bon usage des cautionnements», dans Cautionnements et Assurances, Protection contre les risques des projets de construction, The Canadian Institute, le 28 octobre 1987, p. A-6.

Dans le domaine résidentiel, les propriétaires étant des particuliers moins informés, certains constructeurs de maisons unifamiliales et d'immeubles privés opèrent sans contrat de cautionnement.

7. Larticle 1688 du Code civil du Bas Canada (C.c.) se lit comme suit:

«Si l'édifice périt en tout ou en partie dans les cinq ans, par le vice de la construction ou même par le vice du sol, l'architecte qui surveille l'ouvrage et l'entrepreneur sont responsables de la perte conjointement et solidairement."

8. Les tribunaux ont étendu l'application de 1688 C.c. aux ingénieurs, ces derniers étant appelés à surveiller les travaux au même titre que les architectes. «La profession d'architecte et celle d'ingénieur ne faisaient qu'une autrefois et, quoique l'ingénieur ne soit 
bâtiment causée par un vice de construction ou un vice de sol. La preuve de la négligence n'est pas requise puisque, à cet égard, la loi édicte une présomption de responsabilité; par contre, pour que la malfaçon soit génératrice de réparation, elle doit être susceptible d'entraîner la perte ou ruine du bâtiment ${ }^{10}$. Il s'agit d'une responsabilité légale ${ }^{11}$, d'ordre public ${ }^{12}$, qui est encourue que le lien juridique soit contractuel ou non. Plusieurs auteurs soutiennent que l'article 1688 C.c. fonde une responsabilité contractuelle ${ }^{13}$. Selon la théorie de l'accessoire au contrat, le recours serait transmis aux tiersacquéreurs ou au propriétaire qui n'a pas lui-même contracté avec l'architec$\mathrm{te}^{14}$. Quel que soit la nature du lien juridique qui le lie à l'entrepreneur, l'architecte ou l'ingénieur, le maître d'ouvrage a un recours en responsabilité en vertu de l'article 1688 C.c.

pas mentionné dans les art. 1688 et 1689 , les deux professions sont maintenant assimilées l'une à l'autre pour les fins de ces dispositions": Desgagné c. Fabrique de St-Philippe d'Arvida, [1984] 1 R.C.S, 19, 46. La Cour suprême cite, entre autres, l'arrêt Canadian Electric Light Co. c. Pringle, (1920) 29 B.R. 26.

9. Turcotte c. Lavoie, [1950] B.R. 161; Louis O. PÉLOQUIN, «La nature juridique de la responsabilité des architectes et entrepreneurs», (1984) $44 R . d u$ B. 775, 790.

10. Léon FARIBAULT, Traité de droit civil du Québec, Montréal, Wilson \& Lafleur Itée, tome 12 Du louage, p. 439, cité dans: Donolo Inc. c. St Michel Realties Inc., [1971] C.A. $536,541$.

11. Canadian Consolidated Rubber Co. c. Pringle, [1930] R.C.S. 477; Hill-Clarke-Francis Ltd. c. Northland Groceries (Quebec) Ltd., [1941] R.C.S. 437.

12. L. O. PÉLOQUIN, loc. cit., note 9, 786-791; Fabrique de la Paroisse St-Philippe d'Arvida c. Desgagné, [1979] C.A. 198, 205, conf. par [1984] 1 R.C.S. 19.

13. L. O. PÉLOQUUIN, loc. cit., note 9, 793; Thérèse ROUSSEAU-HOULE, Les contrats de construction en droit public et privé, Montréal, Wilson \& Lafleur, 1982, pp. 412-413. On a interprété ainsi les propos de la Cour d'appel dans l'affaire Fabrique de la Paroisse St-Philippe d'Arvida c. Desgagné, précitée, note 12, alors que la Cour a en réalité préservé le recours de droit commun. D'ailleurs, la Cour suprême a clairement écrit: «...la responsabilité imposée par les art. 1688 et 1689 à l'architecte et à l'ingénieur est une responsabilité établie par la loi et qui ne dépend aucunement d'un contrat.» précité, note $8,46$.

14. General Motors Products of Canada Ltd c. Kravitz, [1979] 1 R.C.S. 790; L. O. PÉLOQUIN, loc. cit., note 9, 794. 
Le propriétaire conserve aussi ses autres recours ${ }^{15}$, contractuels ${ }^{16}$ ou délictuels ${ }^{17}$, lorsque l'édifice n'est pas menacé de ruine, donc en dehors de l'application de 1688 C.c. L'entrepreneur sera responsable des problèmes découlant d'un ouvrage non satisfaisant, soit en raison d'une malfaçon ou du non-parachèvement. Il est reconnu que la réception des travaux par le maître d'ouvrage libère les constructeurs de leur responsabilité à l'égard des vices apparents, mais non à l'égard des vices cachés ${ }^{18}$, ces derniers seront alors générateurs de responsabilité. Si le défaut de construction est dû à une erreur de conception ou de dessin attribuable à l'architecte ou à l'ingénieur, c'est ce professionnel qui aura à supporter les coûts de la réparation.

Quant au dernier fondement de responsabilité, soit la garantie légale contre les vices cachés ${ }^{19}$, seul le propriétaire y est tenu. Par conséquent, un acheteur pourra poursuivre le constructeur-propriétaire en diminution de prix ou en résolution de la vente ${ }^{20}$.

\section{PARTIE I - LES ASSURANCES CONTRE LES MALFAÇONS}

\section{A - Les diverses assurances de responsabilité}

Le Code civil du Bas Canada prévoit qu'une personne peut contracter une assurance pour se protéger contre les conséquences pouvant découler de sa propre responsabilité civile, contractuelle ou extracontractuelle ${ }^{21}$. De plus, tous les intervenants possèdent un intérêt d'assurance sur les biens se trouvant sur le chantier ${ }^{22}$ ainsi que dans leur propre responsabilité civile ${ }^{23}$.

15. Turcotte c. Lavoie, précité, note 9; Fabrique de la Paroisse St-Philippe d'Arvida c. Desgagné, précité, note 12.

16. Art. 1065 C.c.

17. Art. 1053 C.c.; Fitzpatrick Ltd. c. Brett, [1969] C.S. 144.

18. Turcotte c. Lavoie, précité, note 9; Duelz c. Kajandi, [1960] C.S. 89.

19. Art. 1522 C.c.

20. Art. 1526 C.c.

21. Art. 2475 et 2600 C.c.

22. Art. 2580 C.c.; Commonwealth Construction c. Imperial Oil, [1978] 1 R.C.S. 317.

23. Didier LLUELLES, Droit des assurances - Aspects contractuels, Montréal, Les Éditions Thémis Inc., 1986, p. 244. 
Ces contrats sont en général inspirés des modèles américains (CGL américain) et la même formule est employée partout au Canada ${ }^{24}$. À première vue, la chose peut surprendre étant donné que le droit des assurances est régi par le droit privé et l'est, par conséquent, par le droit civil ${ }^{25}$. Toutefois, le droit des assurances a cette particularité de prendre ses sources à la fois dans le droit anglais, à cause de ses origines maritimes, et dans le droit français, en tant que droit privée ${ }^{26}$. De plus, l'industrie de l'assurance a ses exigences $^{27}$ qui produisent leurs effets sur tout le continent américain, à tel point que même les tribunaux supérieurs reconnaissent que le droit des assurances québécois ne peut pas faire abstraction du contexte juridique général canadien ${ }^{28}$. En conséquence, la rédaction et l'interprétation des contrats d'assurance subiront l'influence du droit canadien, tout en gardant leur particularité québécoise.

Nous verrons successivement le cas des architectes et des ingénieurs, et par la suite celui des entrepreneurs.

24. D'ailleurs, le Bureau d'assurances du Canada (BAC) rédige des modèles de polices de toutes sortes qui s'appliquent uniformément à travers le Canada.

25. LActe de Québec de $\mathbf{1 7 7 4}$ a réintroduit le droit civil pour gouverner les affaires privées au Bas-Canada, régime qui avait été mis de côté lors de la conquête de la NouvelleFrance par l'Angleterre en 1763. Cependant, le droit public relève de la Common Law: Acte qui règle plus solidement le Gouvernement de la Province de Québec dans l'Amérique Septentrionale (R.-U.), 1774, 14 Geo. III, c. 83, art. VII, IX et XI; S.R.C. 1985, App. II, $\mathrm{n}^{\circ} 2$.

26. D. LLUELLES, op. cit., note 23, pp. 9-10; Aetna Casualty and Surety Co. c. Le Groupe Estrie Mutuelle d'Assurance contre l'incendie, [1990] R.J.Q. 1795 (C.A.).

27. En effet, «les règles sur l'assurance doivent présenter une certaine uniformité avec les autres provinces, si l'on veut assurer le développement concurrentiel de nos entreprises et protéger adéquatement le public.» Introduction du Projet de loi 7 , notes explicatives de la Loi sur les assurances, Québec, 1973.

28. Banque nationale de Grèce c. Katsikonouris, [1990] 2 R.C.S. 1029; Caisse populaire des Deux Rives c. Société mutuelle d'assurance contre l'incendie de la Vallée du Richelieu, [1990] 2 R.C.S. 995. 


\section{$1 \quad$ - Les architectes et les ingénieurs}

Les architectes et les ingénieurs sont des professionnels ${ }^{29}$ qui, à ce titre, peuvent souscrire à des assurances de responsabilité couvrant leurs gestes professionnels. Ces polices d'assurance prévoient une protection pour tous les actes posés par l'assuré: plan, devis, conception etc..., ainsi que pour la responsabilité encourue par la surveillance des travaux, selon les besoins de l'assuré. Tous les domaines d'activités de ces professionnels sont donc sujets à une couverture, laquelle devra produire ses effets, à moins de clauses d'exclusion au contrat ou d'un motif de nullité ou de déchéance du contrat ${ }^{30}$. Ainsi, les réclamations pour vices cachés ou malfaçons résultant d'un geste fautif de l'architecte ou de l'ingénieur seront en général couvertes par le contrat d'assurance.

Les primes d'assurances sont fixées en fonction du risque et de la mutualité $e^{31}$. Le marché d'assurance québécois, économie et géographie obligent, est intimement lié au reste du Canada et aux États-Unis ${ }^{32}$, de sorte que le taux de prime ne reflète pas nécessairement les risques inhérents aux architectes et ingénieurs québécois ${ }^{33}$. L'inflation, les réclamations de plus en plus nombreuses ainsi que les montants élevés accordés aux victimes par les tribunaux sont autant de facteurs qui ont contribué à l'augmentation des primes et entraîné des franchises importantes ${ }^{34}$.

29. Ces professions sont régies par le Code des professions, précité, note 5, ainsi que par la Loi sur les architectes, précitée, note 3 et la Loi sur les ingénieurs, précitée, note 4.

30. Tels la déclaration initiale de risque incomplète ou frauduleuse: art. 2485 C.c., la faute intentionnelle de l'assuré: art. 2563; al. 2 C.c.

31. D. LLUELLES, op. cit., note 23, p. 7.

32. Bernard FARIBAULT, «Du papillon à la chrysalide ou l'étrange métamorphose de l'assurance de responsabilité», (1987) 55 Assurances 300.

33. En effet; le marché québécois est trop restreint pour être évalué séparément de ses voisins, il subit donc leur influence sinon celle du marché mondial: Claude BARRY, «Gestion des risques et assurance de responsabilité pour architectes et ingénieurs», dans Cautionnements et Assurances, Protection contre les risques des projets de construction, op. cit., note 6 ; pp. F-1 et F-2.

34. Id, pp. F-6 à F-10. 
Malgré tous ces problèmes, l'assurance de responsabilité demeure la protection la plus adéquate contre les risques rattachés à l'exercice de ces professions. Même en l'absence d'obligation légale d'assurance, il est reconnu que bien peu sinon aucun professionnel n'oserait exercer sans avoir préalablement souscrit un tel contrat.

\section{$2 \quad-\quad$ Les entrepreneurs}

\section{- les types de contrat}

Plusieurs types de contrats d'assurance sont offerts aux entrepreneurs. Ceux qui nous intéressent concernent principalement les accidents de parcours pouvant survenir pendant la construction.

Le contrat d'assurance de responsabilité civile générale couvre les dommages corporels et matériels résultant d'une cause externe ou d'un accident $^{35}$. Le montant d'assurance sera déterminé selon les besoins de l'assuré et la prime sera évaluée compte tenu de ce montant, du risque ainsi que de l'importance du chantier. Ce contrat produira des effets au-delà de sa durée, car il prévoit que les dommages seront indemnisés «...pour autant qu'ils soient occasionnés par un accident survenant en cours de contrat.» Dans ces circonstances, l'assureur cocontractant sera tenu de respecter ses engagements, même pour les dommages apparaissant après la terminaison du contrat. En effet, la prescription d'un recours pour dommages corporels est d'une année ${ }^{36}$, et celle pour dommages matériels de deux ans ${ }^{37}$ à compter de l'événement.

35. Une clause générale d'un contrat d'assurance de responsabilité générale offert aux entrepreneurs peut se lire comme suit (Formule du Bureau d'assurance du Canada): «L'assureur garantit l'assuré contre les conséquences pécuniaires de la responsabilité civile pouvant incomber à l'assuré en raison:

- de dommages corporels survenant au cours du présent contrat;

- de dommages matériels survenant au cours du présent contrat du fait d'un accident, de privation de jouissance des biens atteints par lesdits dommages, ou de privation de jouissance de biens corporels non endommagés pour autant qu'elle soit occasionnée par

36. Art. 2262 C.c.

37. Art. 2261 C.c. 
L'assurance des chantiers, quant à elle, garantira les biens se trouvant sur les lieux de la construction. Cette couverture est établie en fonction de la valeur réelle de l'immeuble au début des travaux, des caractéristiques de la construction et de la valeur estimée de l'immeuble à la fin des travaux. Cette garantie a une portée limitée et ne produira ses effets que durant les travaux, ces derniers étant l'objet même de la protection ${ }^{38}$.

Par ailleurs, plusieurs couvertures peuvent être offertes, par un même assureur, dans une même police globale «wrap up» moyennant une prime unique $^{39}$. Cette police a l'avantage de couvrir à la fois tous les intervenants sur un chantier de construction: entrepreneur, sous-entrepreneur, architecte, ingénieur, etc., qui, au lieu de prendre chacun une assurance pour un montant déterminé et visant des objets déterminés, mettent leurs intérêts en commun et souscrivent un seul contrat.

Le problème de l'intérêt d'assurance des divers participants s'est posé. À prime abord, tous les hommes de métier se trouvant sur un chantier n'ont pas l'intérêt d'assurance dans tous les biens qui s'y trouvent. Cependant, toutes les parties ont un intérêt commun, l'achèvement des travaux et l'intérêt assurable se trouve justement dans la poursuite de ce but, celui-ci étant l'objet de leurs contrats respectifs ${ }^{40}$. Chacun ayant l'intérêt d'assurance requis pour

38. Une clause usuelle concernant l'étendue de la protection est ainsi libellée (Formule du Bureau d'assurance du Canada):

«Dans les limites du présent contrat, la présente assurance produit ses effets sur les biens se trouvant sur le chantier, depuis leur prise en charge par l'assuré à la suite de leur déchargement, jusqu'à l'expiration d'une période de trente jours suivant la fin des travaux. La présente assurance prend fin, en ce qui concerne toute partie du chantier, dès la mise en service ou l'occupation à des fins autres que de construction, d'habitation, de bureau, ou d'installation, de vérification ou de remisage de matériel, d'équipement ou de machines.»

39. Alain LÉTOURNEAU, «L'assurance en matière de construction», dans Cautionnements et Assurances, Protection contre les risques des projets de construction, op. cit., note 6, p. D-6.

40. Commonwealth Construction c. Imperial Oil, précité, note 22, 323-324; voir aussi Scott c. Wawanesa Mutual Insurance Co., [1989] 1 R.C.S. 1445 sur la question des intérêts multiples. 
l'ensemble du chantier, il devient «assuré» au sens de la police d'assurance.

Un principe fondamental veut qu'un assureur ne soit pas subrogé contre l'assuré lui-même ${ }^{41}$, ce dernier ne pouvant être tenu responsable de la négligence de ses ouvriers face à son propre assureur ${ }^{42}$. Un dommage causé par un des participants à la police globale privera l'assureur de la subrogation, un facteur qui devrait influer sur le taux de prime!

\section{- les exclusions}

Tant les polices d'assurance de responsabilité que les polices d'assurance des chantiers comportent des dispositions visant à exclure de la couverture les malfaçons dues à des défauts causés par la main-d'oeuvre «faulty workmanship» ${ }^{43}$ ou à la conception des plans et des dessins. Ces clauses sont rédigées différemment selon les assureurs, mais le fondement reste le même $e^{44}$. Elles ont reçu une interprétation large, même en l'absence de preuve de négligence de la part des ouvriers $^{45}$, de sorte qu'on peut se demander jusqu'à quel point l'objet même du contrat n'aurait pas été évacué.

41. Commonwealth Construction c. Imperial Oil, précité, note 22, 321; art. 2576 C.c.

42. Id, 322, la Cour suprême s'appuie sur l'affaire Agnew-Surpass Shoe Stores Ltd. c. Cummer-Yonge Investments. Ltd., [1976] 2 R.C.S. 221, 251.

43. L'expression «faulty workmanship» est consacré dans le domaine de l'assurance de la construction, à tel point que les tribunaux se sont demandé si une traduction satisfaisante existait: Commercial Union cie d'assurance du Canada c. Pentagon Construction Canada Inc., [1989] R.J.Q. 1399, 1404-1405 (C.A.).

44. Une clause d'exclusion est ainsi libellée:

«Sont exclus:

Les frais inhérents à la bonne exécution des travaux, et rendus nécessaires par des défauts dans:

les matériaux, leur emploi ou leur choix;

la main-d'oeuvre;

les plans ou la conception;

étant précisé que l'assurance produit néanmoins ses effets en ce qui concerne les sinistres entraînés par voie de conséquence et n'étant pas exclus par ailleurs.»

(Extrait d'une police d'assurance des chantiers, formule du Bureau d'assurance du Canada)

45. Eric A. DOLDEN, «All Risk Builders Risk Policies Emerging Trends», 9 Can. L.J. 20, 22-23; Pentagon Construction (1969) Co. Ltd. c. United States Fidelity and Guarantee Co., [1977] 1.L.R. 674 (B.C. C.A.); Bird Construction Co. c. United States Fire Ins. Co., (1987) 18 C.C.L.I. 92 (Sask. C.A.). 
Ces exclusions relatives aux défauts résultant de la main-d'oeuvre et des plans ou de la conception se retrouvent aussi dans les polices globales, lesquelles peuvent être illusoires dans certains cas. Par exemple, un architecte assuré s'est vu refuser le bénéfice de l'assurance globale car le fait générateur venait d'une erreur dans ses plans ${ }^{46}$. Il s'agissait en fait d'un litige entre l'assureur de responsabilité de l'architecte et l'assureur des chantiers. Le tribunal a décidé que la police d'assurance des chantiers ne pouvait pas s'appliquer en raison de l'exclusion ${ }^{47}$.

En conséquence de ces exclusions au contrat d'assurance, les malfaçons et vices cachés sont à la charge de l'entrepreneur, que sa responsabilité découle du contrat de construction, de son obligation de diligence ou du régime de l'article 1688 C.c.

\section{- le tempérament à ces exclusions}

Les contrats prévoient le plus souvent un tempérament à ces exclusions que nous appellerons «l'exception à l'exclusion». En effet, les défauts causés par la main-d'oeuvre ou les déficiences suite à un plan ou une conception erronés sont exclus. Par contre, seront couverts les dommages aux biens qu'ont entraîné ces défauts. Il y a lieu d'examiner quels dommages seront alors couverts grâce à cette «exception à l'exclusion».

De la même façon que les tribunaux ont interprété les clauses d'exclusion largement à l'avantage des assureurs, ils ont donné une réception restrictive à l'exception et ont été réticents à en accorder le bénéfice à l'assuré lorsque le bien endommagé n'appartenait pas à un tiers ${ }^{48}$. Ainsi, lorsque le recouvrement des fils d'une installation électrique est défectueux, les dommages causés par l'eau à l'installation seront exclus, mais le seront aussi les coûts qu'a entraînés la panne, dommages que l'on pourrait qualifier de

46. Simcoe \& Erie General Insurance Co. c. Royal Insurance Co. of Canada, [1983] I.L.R. 6149 (Alta C.A.).

47. Id.

48. E. A. DOLDEN, loc. cit., note 45, 27; Bird Construction c. United States Fire Ins. Co., précité, note 45; Golden Eagle Canada Ltd. c. The American Home Assurance Co., [1978] C.S. 699. 
subséquents, donc devant tomber sous le coup de l'exception ${ }^{49}$. Un défaut dans le plan de la structure d'un pont pouvant causer son écroulement est aussi exclu de la couverture, même si l'erreur dans le plan n'affecte qu'une partie de l'ouvrage, cette partie étant indissociable de l'ensemble de la construction ${ }^{50}$. Toutefois, lorsque l'on peut séparer nettement les causes de dommages, soit d'une part les dommages causés par l'acte fautif (exclu) et ceux résultant de l'objet défectueux (exception à l'exclusion) ces derniers seront alors dédommagés par l'assureur ${ }^{51}$.

Dans un récent jugement, la Cour d'appel du Québec a fait preuve de plus d'ouverture dans l'interprétation des clauses d'exclusions et de leurs exceptions $^{52}$. Il s'agissait de déterminer si les dommages causés à un pilier soutenant un pont étaient couverts par le contrat d'assurance des chantiers. La Cour refait l'étude de la jurisprudence ayant cours au Canada et fait les distinctions qui s'imposent. D'une part, elle affirme qu'il ne peut être donné lieu à l'exclusion de «faulty workmanship» sans preuve de négligence de la part des ouvriers et que c'est l'assureur qui a le fardeau d'établir la faute ${ }^{53}$, sinon, on considérera qu'il s'agit d'un accident, événement couvert par le contrat $^{54}$. D'autre part, la Cour émet des commentaires quant à l'exception à l'exclusion. Elle affirme qu'en présence d'une exception à la clause d'exclusion, il fallait évaluer les conséquences de la faute de la main-d'oeuvre et donner effet à l'exception. Rejetant les solutions jurisprudentielles des autres provinces, la Cour a clairement établi que la clause d'exclusion produira ses effets sur le résultat immédiat de la malfaçon, c'est-à-dire le dommage causé à la pièce même affectée du vice, et non aux dommages découlant du défaut de la pièce ${ }^{55}$. Cette interprétation du contrat d'assurance est, à notre avis,

49. Sayers \& Associates Limited c. The Insurance Corporation of Ireland, [1981] I.L.R. 5476 (Ont. C.A.).

50. Simcoe \& Erie General Insurance Co. c. Royal Insurance Co. of Canada, précité, note 46.

51. Poole-Prichard Canadian Limited c. The Undenwriting Members of LLoyds, [1970] I.L.R. 915 (Alta S.C.).

52. Commercial Union Cie d'assurance du Canada c. Pentagon Construction Canada Inc., précité, note 43.

53. Id, 1412.

54. Id, 1403.

55. Id, 1405 . 
plus conforme au droit et fait échec à la non responsabilité des assureurs dans le domaine de la construction. Est-ce l'effet du hasard ou est-ce parce que l'esprit du droit des assurances a pris un virage avantageant les assurés depuis la réforme de la loi en $1976^{56}$, qu'une telle décision émane d'un tribunal québécois?

\section{B - - Critique des protections d'assurance}

On peut penser qu'un entrepreneur ayant pris soin de contracter des assurances bénéficie de bonnes protections. Ceci n'est juste qu'en ce qui concerne les dommages consécutifs à un accident, car nous avons vu que dans la plupart des polices le risque de malfaçon est exclu. En l'absence de définition dans le contrat, les tribunaux ont donné au terme «accident» son sens usuel en regard du contexte du contrat ${ }^{57}$, c'est-à-dire «...un événement involontaire ou imprévu, soit dans l'événement lui-même, soit dans ses effets» ${ }^{58}$. Il s'agit «...d'une mésaventure ou d'une malchance imprévue» ${ }^{59}$.

À ce titre, les erreurs de conception et de fabrication dues à une faute non intentionnelle répondent à cette définition et devraient être couvertes par le contrat d'assurance, à moins d'une d'exclusion expresse au contrat ${ }^{60}$. De même, est un dommage accidentel celui qui découle de la malfaçon. Lorsque la construction viciée entraîne un dommage à un autre bien, il s'agit alors d'un accident qui devrait être considéré comme un événement couvert par le contrat, sans besoin de stipulation expresse à cet effet. Les clauses «d'exception à l'exclusion» ne seraient donc pas nécessaires.

56. L.Q. 1974, c. 70. En effet, plusieurs dispositions de la loi sont maintenant d'ordre public, obligeant ainsi les assureurs à respecter certaines limites dans les exclusions.

57. Travelers du Canada c. Entreprises Cotenor Ltée, [1978] C.A. 17, 17-18; voir: JeanPierre LUSIGNAN, «L'accident ou la faute non intentionnelle en droit des assurances privées québécois», (1990) C. de D. 155-189.

58. Travelers du Canada c. Entreprises Cotenor Ltée, précite, note 57.

59. Canadian Indemnity Co. c. Walkem Machinery \& Equipment Ltd., [1976] 1 R.C.S. 309, 316: la Cour suprême y a interprété le terme «accident».

60. Groupe Desjardins, Assurances générales c. L. Breton \& Fills (La Sarre) Ltée, précité, note 1,225 . 
Le législateur a prescrit que la simple faute ou négligence de l'assuré doit être expressément et limitativement stipulée au contrat ${ }^{61}$, cet article étant d'ordre public relatif, on ne peut y déroger qu'en faveur du preneur ${ }^{62}$. Si c'est la faute ou la négligence qui est la cause de la malfaçon, l'exclusion devrait être précisée. Or, le libellé des exclusions utilisé est habituellement laconique: «sont exclus les frais... rendus nécessaires par des défauts dans... la main-d'oeuvre», on ne peut pas conclure qu'il s'agit d'une «stipulation limitativement et expressément énoncée au contrat». À notre avis, ces exclusions ne répondent pas aux exigences impératives de la loi, elles sont donc illégales ${ }^{63}$.

L'interprétation que les tribunaux canadiens ont donné à l'exclusion de «faulty workmanship» est critiquable sur deux aspects. D'une part, ce type de clause ne devrait pas produire d'effet car elle ne respecte pas la loi ${ }^{64}$. D'autre part, présumer de la faute ou de la négligence des ouvriers écarte la thèse de l'accident, élément couvert par le contrat. Sur ce point, nous partageons l'avis de la Cour d'appel du Québec lorsqu'elle exige de l'assureur qu'il fasse la preuve de la faute ou de la négligence pour appliquer l'exclusion, à défaut de quoi on conclura que les malfaçons ont été causées par accident ${ }^{65}$. Interpréter autrement constitue une lecture purement mécanique étrangère aux règles d'interprétation. La Cour suprême ${ }^{66}$ nous a enseigné les règles à suivre dans l'interprétation des contrats d'assurance. Il faut, en premier lieu, rechercher

61. Art. 2563, al. 1 C.c.

62. Art. 2500 , al. 2 C.c.

63. L'article 2563 , al. 1 C.c. a donné lieu à peu d'interprétation. Voir: Jean-Guy BERGERON, Les contrats d'assurance, lignes et entre-lignes, tome 1, Sherbrooke, Les Éditions SEM inc, 1989, pp. 252-260. Jean-Guy BERGERON, «Quelques points de rencontre entre la responsabilité civile et l'assurance», dans Responsabilité civile et les dommages: en constante évolution, The Canadian Institute, le 7 décembre 1990, p. 9.

64. Comme nous venons de le démontrer, lorsqu'une clause du contrat va à l'encontre de l'ordre public, cette clause est «sans effet dans la mesure de la contradiction» Lejeune c. Cumis Insurance Society Inc., [1989] 2 R.C.S. 1048, 1061.

65. Commercial Union cie d'assurance du Canada c. Pentagon Construction Canada Inc., précité, note 43.

66. Exportations Consolidated Bathurst Ltée c. Mutual Boiler and Machinery Insurance Co., [1980] 1 R.C.S. 888. 
l'intention véritable des parties ${ }^{67}$, et ce n'est qu'en cas d'ambiguïté que l'on recourt aux autres règles.

L'objet même du contrat d'assurance est de protéger l'assuré contre les événements les plus susceptibles de porter atteinte à son patrimoine ${ }^{68}$ et en matière de construction, les accidents inhérents aux chantiers sont particulièrement coûteux. Le contrat doit donc être interprété de façon à donner effet au contrat plutôt que l'inverse ${ }^{69}$ : Ce qui est visé par l'exclusion est un comportement fautif, qui doit être spécifié et dont on doit faire la preuve.

Il est donc aisé de soutenir que l'interprétation des exclusions doit être réexaminée à la lumière des principes élaborés par les tribunaux en matière d'interprétation des contrats d'assurance ${ }^{70}$ ainsi que des exigences du Code civil $^{71}$. Lorsque des clauses sont libellées de façon à exclure de la couverture les dommages résultant d'un défaut de la main-d'oeuvre, il faut considérer que ces défauts doivent résulter d'une faute, sinon le dommage sera couvert par le contrat. Si la malfaçon est causée par une faute, les tribunaux devront évaluer l'exclusion en regard de l'article 2563 , al. 1 C.c.

Ces exclusions ne sont pas, selon nous, applicables dans le cas d'un accident et leur légalité est douteuse à l'égard de la simple faute. Un tel point de vue, il est vrai, rompt avec la compréhension traditionnelle de l'assurance de responsabilité contre les malfaçons, mais le contenu et la forme des polices d'assurance peut s'adapter aux besoins exprimés par les assurés ${ }^{72}$.

Une constatation s'impose, l'assurance n'est que de peu d'utilité pour les entrepreneurs comme mode de protection contre les malfaçons. Que ce soit parce que l'assurance est inefficace ou par pratique traditionnelle, le

67. Id. 901.

68. Id, 903 .

69. Id, 901 et 902; Hadley Shiping Co. c. Eagle Star Insurance Co. of Canada, J.E. 80-566 (C.S.).

70. Exportations Consolidated Bathurst Ltée c. Mutual Boiler and Machinery Insurance Co., précité, note 66; Scott c. Wawanesa Mutual Insurance Co., précité, note 40.

71. Art. 2563 , al. 1 C.c.

72. J.-G. BERGERON, op. cit., note 63, p. 258. 
cautionnement est privilégié par l'industrie de la construction en tant que façon de se garantir contre les pertes que peuvent encourir un tel risque.

\section{PARTIE II - LES CAUTIONNEMENTS}

\section{A - Les cautionnements en tant que garanties contre les malfaçons}

Le cautionnement est «une sûreté personnelle qui îacilite l'exécution de l'obligation ${ }^{73}{ }$. Ce n'est pas un régime qui rajoute un débiteur solidaire, à moins d'un engagement en ce sens de la part de la caution ${ }^{74}$, ni qui relève le débiteur de son obligation, mais c'est un «...acte par lequel une personne s'engage à remplir l'obligation d'une autre pour le cas où celle-ci ne la remplirait pas. ${ }^{75}$ Le contrat de cautionnement est accessoire au contrat principal $^{76}$. À ce titre, la caution poursuivie avant le débiteur principal peut opposer au créancier le bénéfice de discussion; ce n'est qu'advenant la preuve de l'insolvabilité du débiteur que la caution est tenue de payer ${ }^{77}$. Ce paiement ne libère pas pour autant le débiteur car la caution garde contre ce dernier un recours pour se faire rembourser ${ }^{78}$. Le cautionnement est donc une institution à l'avantage du créancier de l'obligation et non à l'avantage du débiteur. Il a par contre la vertu d'attester de la compétence et de la solvabilité de l'entrepreneur, les compagnies de garantie enquêtant avant de s'obliger.

Le cautionnement est largement utilisé dans le domaine de la construction, à diverses étapes du contrat. On trouve le cautionnement de soumission, qui garantit la conclusion du contrat, le cautionnement d'exécution du contrat ainsi que le cautionnement de paiement des matériaux et de la

73. Louise POUDRIER-LEBEL, Le cautionnement par compagnie de garantie, Montréal, Les Éditions Yvon Blais Inc., 1986, p. 189.

74. Art. 1941 C.c.

75. Art. 1929 C.c.

76. Pierre CIOTOLA, Droit des sûretés, $2^{\star}$ éd., Montréal, Les Éditions Thémis Inc., 1987, p. 43 .

77. Id, p. 43; art. 1941 C.c.

78. La caution procédera par action personnelle (art. 1948 C.c.) ou de type subrogatoire, article 1950 C.c. Voir la discussion de P. CIOTOLA, op. cit., note 76, pp. 47-49. 
main-d'oeuvre ${ }^{79}$. Nous nous arrêterons au cautionnement d'exécution de contrat, car c'est celui qui garantit au maître d'ouvrage l'exécution complète et satisfaisante de la construction qu'il a financée. Ce type de cautionnement complète les couvertures offertes dans les contrats d'assurance de responsabilité et d'assurance des chantiers.

Étant donné qu'il s'agit généralement d'un contrat formé par la volonté des parties ${ }^{80}$, il en sera la loi. Il est donc important d'attacher une attention particulière à la rédaction de ses clauses. Le cautionnement d'exécution englobe habituellement l'obligation de compléter les travaux ainsi que celle de réparer les malfaçons ${ }^{81}$.

Dans la plupart des contrats, la caution s'engage à garantir la reprise et le parachèvement des travaux, à défaut du respect de ses obligations par l'entrepreneur. L'on peut penser que ce dernier, s'il a été incapable de mener

79. L. POUDRIER-LEBEL, loc. cit., note 6, A-5.

80. Pour fins de discussion, nous faisons abstraction des obligations relevant des législations concernant les travaux publics qui obligent «...le ministre... à obtenir, pour Sa Majesté, une sûreté valable - libellée au nom de celle-ci - garantissant l'exécution régulière de ces travaux...»: Loi sur les travaux publics, précitée, note 6, art. 16.

81. L. POUDRIER-LEBEL, loc. cit., note 6, A-27. Voici un extrait d'un contrat de cautionnement à l'avantage de l'acheteur d'une maison neuve (contrat de cautionnement offert par L'association de la construction du Québec)

«Par les présentes, le Plan (sic) aux conditions ci-après contenues se porte caution des obligations de la personne désignée comme l'entrepreneur au présent certificat, mais uniquement:

1.1 À l'égard de la reprise ou du parachèvement des travaux inscrits à l'attestation d'achèvement et de possession signée par l'entrepreneur et par l'acheteur...

1.2 À l'égard des vices cachés et dénoncés par écrit au Plan dans l'année suivant la signature de l'attestation d'achèvement et de possession, vices cachés signifiant pour les fins des présentes les vices qu'un acheteur raisonnablement diligent ne pouvait déceler au moment de l'émission de l'attestation d'achèvement et de possession et qui sont de la nature de ceux qu'un vendeur est tenu de garantir conformément à l'article 1522 du Code civil.

1.3. À l'égard des vices de construction ou vices du sol apparus et dénoncés par écrit au Plan, dans les 5 ans suivant l'émission de l'attestation d'achèvement et de possession, signifiant ceux ayant entraîné ou risquant d'entraîner la perte partielle ou totale du bâtiment résidentiel et qui sont visés à l'article 1688 du Code civil de la provincè de Québec.». 
le projet à terme, doit être dans une bien mauvaise situation financière, de sorte que le recours de la caution pour le recouvrement de ses débours pourrait être illusoire.

La compagnie de garantie cautionnera aussi les dépenses occasionnées par les vices cachés, entendus au sens de l'article 1522 C.c., c'est-à-dire ceux qui rendent impropres à l'usage auquel on le destinait l'objet de la construction ou qui en diminuent tellement l'utilité que le prix en serait diminué. Il s'agit ici de défauts graves, qui n'étaient pas apparents lors de l'acceptation des travaux par le maître d'ouvrage ${ }^{82}$. Cette clause est d'application limitée dans le temps, soit d'une ou de deux années après l'acceptation des travaux par le propriétaire.

Quant à la garantie pour les réclamations en vertu de l'article 1688 C.c., elle entrera en jeu lorsque les vices de construction ou de sol peuvent entraîner la perte partielle ou totale de l'édifice. La caution s'oblige habituellement pour cinq ans, respectant ainsi le délai prévu par la loi.

La durée de la garantie peut cependant être nettement insuffisante. En effet, la responsabilité contractuelle de l'entrepreneur se prescrit par trente $a_{n} s^{83}$ et sa responsabilité délictuelle par deux ans ${ }^{84}$. Une clause limitant l'engagement de la caution à une année dans le cas de vices cachés, peut être nettement insuffisante. Le vice caché, qualifié ainsi justement parce qu'il n'est pas apparent, peut être décelé plus d'une année après la réception des travaux. Il en est de même pour les réclamations faites en vertu de l'article 1688 C.c., le recours pouvant être intenté dans les cinq ans de la perte ${ }^{85}$. Le créancier peut se trouver alors dans une situation où l'entrepreneur est insolvable, alors que le contrat avec la caution est expirér.

82. D'ailleurs, lorsque les travaux sont acceptés, le propriétaire ne peut plus se plaindre des vices apparents: Turcotte c. Lavoie, précité, note 9; Duelz c. Kajandi, précité, note 18.

83. Art. 2242 C.c.

84. Art. 2261 C.c.

85. Art. 2259 C.c.

86. La longue prescription trentenaire dans le domaine contractuel est génératrice de plusieurs problèmes. Le Code civil du Québec, L.Q. 1991, c. 64, qui a été sanctionné le 18 décembre 1991 et qui entrera en vigueur par proclamation, prévoit maintenant une 
Ainsi, le maître d'ouvrage et l'acheteur subséquent bénéficient d'une garantie pour les vices cachés et les vices de construction. Il faudra d'abord poursuivre l'entrepreneur, et ce n'est que lorsque ce dernier sera en défaut que le propriétaire pourra se toumer vers la caution ${ }^{87}$. C'est une bien piètre garantie lorsqu'on considère les délais d'une action en justice. Afin de pallier ces inconvénients, certains contrats de cautionnement prévoient que le propriétaire peut s'adresser à la caution après avoir avisé l'entrepreneur. La caution se posera alors comme conciliateur et, en cas d'échec, la poursuite devant les tribunaux sera le seul moyen efficace ${ }^{88}$.

\section{B - Critique du cautionnement en tant que garantie}

Le cautionnement est souvent pratiqué par des compagnies d'assurance et peut être ainsi assimilé à l'assurance par les non-juristes. Certains se sont demandé si le cautionnement contre rémunération n'avait pas pour effet de transformer le régime juridique du contrat ${ }^{89}$ et le transformer en contrat d'assurance. Me Poudrier-Lebel a fait une étude approfondie des ressemblances et différences entre les cautionnements et les contrats d'assurance ${ }^{90}$. Elle arrive à la conclusion que le cautionnement, bien que son objet soit la garantie contre le risque ${ }^{91}$ et malgré son caractère onéreux, est un contrat différent doté de son propre régime juridique ${ }^{92}$.

D'ailleurs, la controverse sur la nature de l'engagement ne pose qu'un problème fictif, les clauses des contrats étant claires à ce sujet. Toutefois, les conséquences pour l'entrepreneur d'être protégé par un contrat de cautionnement ou un contrat d'assurance sont importantes. "Contrairement à ses collègues architectes et ingénieurs, il ne bénéficie pas d'une protection

prescription de trois ans pour les recours personnels: art. 2925.

87. Art. 1929 et 1941 C.c., d'ailleurs, les contrats de cautionnement prévoient presque toujours une clause à cet effet.

88. Le recours à la conciliation peut être rendu obligatoire par contrat avant toute poursuite légale.

89. L. POUDRIER-LEBEL, op. cit., note 73; p. 194.

90. Id. pp. 194-217.

91. Id., p. 194.

92. Id. pp. 209-217. 
d'assurance de responsabilité contre ses erreurs professionnelles. Et pourtant, il devrait y avoir droit au même titre que celui qui engage sa responsabilité par ses plans et dessins, ou par le jeu de l'article 1688 C.c.

On peut constater que le cautionnement, s'il est un instrument utile dans le domaine de la construction, demeure un régime imparfait dans le cas de la responsabilité pour malfaçons et vices cachés. En effet, ce ne sont que les malfaçons qui entraînent la perte de l'immeuble ou le rendent impropres à l'usage qui sont visées par les contrats de cautionnement; les problèmes causés par des malfaçons qui ne tombent pas dans ces catégories sont à l'unique charge de l'entrepreneur. Le maître d'ouvrage peut se retrouver sans protection en cas de défaut de l'entrepreneur. De plus, autre inconvénient, le tiers lésé doit d'abord réclamer du débiteur principal l'exécution de l'obligation avant de s'adresser à la caution ${ }^{93}$, ce qui peut entraîner des retards et des coûts considérables pour le maître d'ouvrage.

Le cautionnement n'est pas une protection pour l'entrepreneur, car ce dernier est toujours le débiteur principal de l'obligation ${ }^{94}$ et la caution conserve un recours contre lui pour les sommes qu'elle a payées ${ }^{95}$. Il s'agit plutôt d'une garantie à l'avantage du maître d'ouvrage, offerte par l'entrepreneur qui en paie le coût d'adhésion. Le coût d'une réparation pour malfaçons peut être très élevé, et même acculer un constructeur à la faillite.

\section{CONCLUSION}

Si les contrats d'assurance sont peu utilisés en tant que garantie contre les malfaçons, c'est que la pratique dans ce domaine donne au cautionnement la place de choix. Il n'est pas de notre propos de critiquer l'emploi général des cautionnements dans l'industrie de la construction: ils sont utiles et nécessaires. Que l'on pense au cautionnement de soumission, au cautionnement de paiement de la main-d'oeuvre et des matériaux et même au cautionnement d'exécution. Nous sommes toutefois convaincue que

93. Voir note 86.

94. Art. 1941 C.c.

95. Art. 1948 et 1950 C.c. 
l'assurance pourrait jouer un grand rôle en ce qui a trait aux erreurs qui surviennent en cours de chantier. Les malfaçons apparaissant pendant la construction ainsi que les vices qui ne sont découverts qu'après la réception des travaux par le propriétaire pourraient ainsi faire l'objet d'une assurance de responsabilité professionnelle.

Au Québec, nous connaissons un système de contrôle visant à assurer la sécurité et la solidité des travaux ${ }^{96}$. Les différents intervenants doivent détenir des licences ${ }^{97}$, cartes de compétence ${ }^{98}$ et autres gages de connaissance du métier ${ }^{99}$ pour oeuvrer dans le domaine, et les constructions ellesmêmes doivent répondre à des normes établies ${ }^{100}$. L'obtention des licences et cartes de compétence ainsi que la conformité des travaux à la loi font l'objet d'inspections sur les chantiers ${ }^{101}$. S'il est un risque assurable, c'est bien celui qui est pratiquement garanti par les exigences administratives de l'exercice du métier. Tous ces contrôles écartent la croyance voulant que l'assurance contre les malfaçons encouragerait la négligence.

96. Voir le texte de Claire MOFFET et Louise VIAU, «La responsabilité pénale des constructeurs en droit canadien et québécois», dans la présente édition. Les auteures nous rapportent que pas moins de vingt lois, sans compter de nombreux règlements, s'appliquent au domaine de la construction.

97. Tous les entrepreneurs doivent détenir une licence pour effectuer des travaux: Loi sur la qualification professionnelle des entrepreneurs de construction, L.R.Q., c. Q-1, quant aux architectes et ingénieurs, ils sont soumis au contrôle de leur corporation professionnelle respective pour obtenir le droit de pratiquer leur profession: Loi sur les architectes, précitée, note 3 , art. 10 et Loi sur les ingénieurs, précitée, note 4 , art. $15,17$.

98. Les ouvriers oeuvrant sur un chantier doivent détenir une carte de compétence: Loi sur les relations du travail, la formation professionnelle et la gestion de la main-d'oeuvre dans l'industrie de la construction, L.R.Q., c. R-20, art. 85.1 à 85.6.

99. Par exemple n'ont droit aux titres de «maître électricien» et de «maître mécanicien en tuyauterie» que ceux qui y sont autorisés par la loi: Loi sur les mâttres électriciens, L.R.Q., c. M-3, Loi sur les maîtres mécaniciens en tuyauterie, L.R.Q., c. M-4.

100. Loi sur les installations électriques, L.R.Q., c. I-13.01; Loi sur les installations de tuyauterie, L.R.Q., c. I-12.1; Code du bâtiment, R.R.Q., 1981, c. S-3, r. 2; Règlement adoptant le Code électrique canadien, R.R.Q., 1981, c. I-13.01, r. 2; Code de plomberie, R.R.Q., 1981, c. I-12.1, r. 1, pour n'en donner que quelques exemples.

101. Les lois qui réglementent l'industrie de la construction prévoient la nomination d'inspecteurs pour assurer le respect de ces lois: C. MOFFET, L. VIAU, loc. cit., note 96. 
Les avantages de l'assurance sur le cautionnement pour l'entrepreneur sont évidents, c'est l'assureur qui paiera la facture. Le contrat d'assurance est cependant soumis à des règles que ne connaît pas le cautionnement. Les causes de nullité des contrats telles l'erreur, la fraude ou la crainte et la lésion $^{102}$ sont certes applicables en droit des assurances, mais elles sont peu utilisées, ce dernier connaissant ses propres causes de nullité des contrats. Ainsi, une déclaration initiale de risque incomplète ou frauduleuse de l'assuré entraîne la nullité du contrat ${ }^{103}$. C'est le preneur qui a l'obligation de déclarer et celle-ci implique la très haute bonne foi de sa part ${ }^{104}$. De plus, il est tenu de déclarer les aggravations de risque qui relèvent de lui et celles qui sont prévues au contrat ${ }^{105}$, à défaut de quoi l'indemnité payable à l'assuré sera diminuée proportionnellement et la résiliation du contrat pourra même être prononcée $e^{106}$.

Des événements en cours de contrat peuvent aussi entraîner la déchéance des droits de l'assuré à l'indemnité. Ainsi, si le dommage est causé par une faute intentionnelle de sa part ${ }^{107}$, de sa négligence grossière ${ }^{108}$ ou résulte de son acte criminel ${ }^{109}$; ou lorsque l'avis de sinistre est donné tardivement $^{110}$ ou que la preuve de perte est mensongère ${ }^{111}$. Dans de telles circonstances, l'entrepreneur devra assumer lui-même les coûts de ses erreurs, finalité semblable à celle du cautionnement. Le tiers qui croyait pouvoir compter sur la solvabilité d'un assureur peut ainsi se retrouver dans une

102. Art. 991 C.c.

103. Art. 2485 et 2487 C.c.

104. D. LLUELLES, op. cit., note 23 , p. 158 . Canadian Indemnity Co. c. Canadian JohnsManville Co., [1990] 2 R.C.S. 549.

105. Art. 2566 C.c.

106. Art. 2566 , al. 4 et 2488 C.c.

107. Art. 2563 , al. 2 C.c.

108. La négligence grossière est apparentée à la faute intentionnelle lorsque l'assuré aurait pu prévoir le caractère inéluctable du dommage: Co-operative Fire and Casualty Co., c. Saindon, [1976] 1 R.C.S. 735.

109. La loi permet expressément d'exclure de la couverture les dommages causés par des agissements qui relèvent d'un acte criminel: article 2481 C.c.

110. Art. 2572 C.c.; Commercial Union Assurance Co. c. Northumberland General Insurance Co., [1990] R.J.Q. 1813 (C.A.).

111. Art. 2574 C.c. 
situation plus mauvaise que celui qui fait affaires avec un entrepreneur nonassuré mais ayant un cautionnement. Il faut par contre se souvenir que ces motifs de non-couverture sont l'essence même du régime de l'assurance, lequel est fondé principalement sur la mutualité et la répartition scientifique des risques ${ }^{112}$.

Malgré ces inconvénients, le maître d'ouvrage et le propriétaire subséquent gagneraient beaucoup de l'assurance. Lorsque l'événement est prévu dans un contrat d'assurance, l'assureur a l'obligation de défendre son assuré $^{113}$ et indemnisera la perte en son lieu et place ${ }^{114}$; de plus, ce tiers peut poursuivre directement l'assureur ${ }^{115}$. On peut y constater une économie de temps et d'argent.

La malfaçon dans le domaine de la construction demeure un risque assurable, et l'assurance est la meilleure protection qui soit, tant pour l'entrepreneur que pour le propriétaire de l'ouvrage. À cet égard, étant donné les sommes importantes qui sont investies lors de la construction du moindre édifice, il serait souhaitable qu'il soit obligatoire pour les architectes, ingénieurs et entrepreneurs de détenir une assurance de responsabilité ainsi que des garanties de solvabilité adéquates avant de se lancer dans un projet, public ou privé. 